
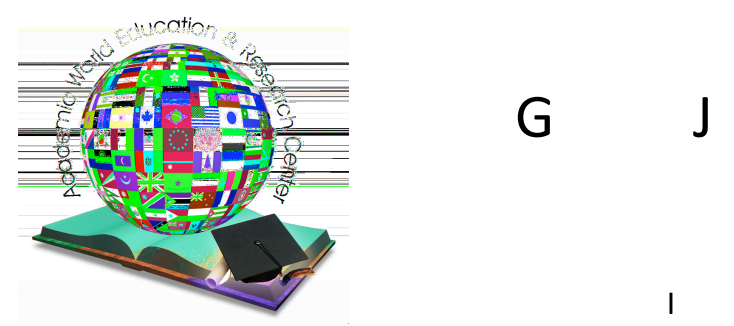

www.awer-center/gjs

\title{
The effect of gender on refusal of suggestion in formal and informal situations among Iranian learners
}

Behzad Rahbar* L Mohammad Reza Oroji L

Fariba Hedayatnejad,

B

Suggested Citation:

B $\mathrm{O} \quad \mathrm{M}$

J

P OCL D A
IA

IA

F L

F

I Global Journal of Sociology 5

M

D

$\mathrm{E}$
Th

C $\mathrm{A}$ $\begin{array}{lll}\text { B } & \text { I } \\ \text { B } & \text { I }\end{array}$

A
C

C

Abstract

$\begin{array}{cccc}N & \text { I } & E F L \\ & C & D C & \end{array}$

।

DC 
$\begin{array}{lllll}B & \mathrm{O} & \mathrm{M} & \mathrm{H} & \mathrm{F}\end{array}$

I Global Journal of Sociology 5

1. Introduction

D

O

A

$\begin{array}{ll}P & P \\ I & E F L\end{array}$

A

\begin{abstract}
$A$
\end{abstract}
A

F B

I

F B

A $\quad B^{G} \quad L^{H}$

A

I

A

A

A

I

A 


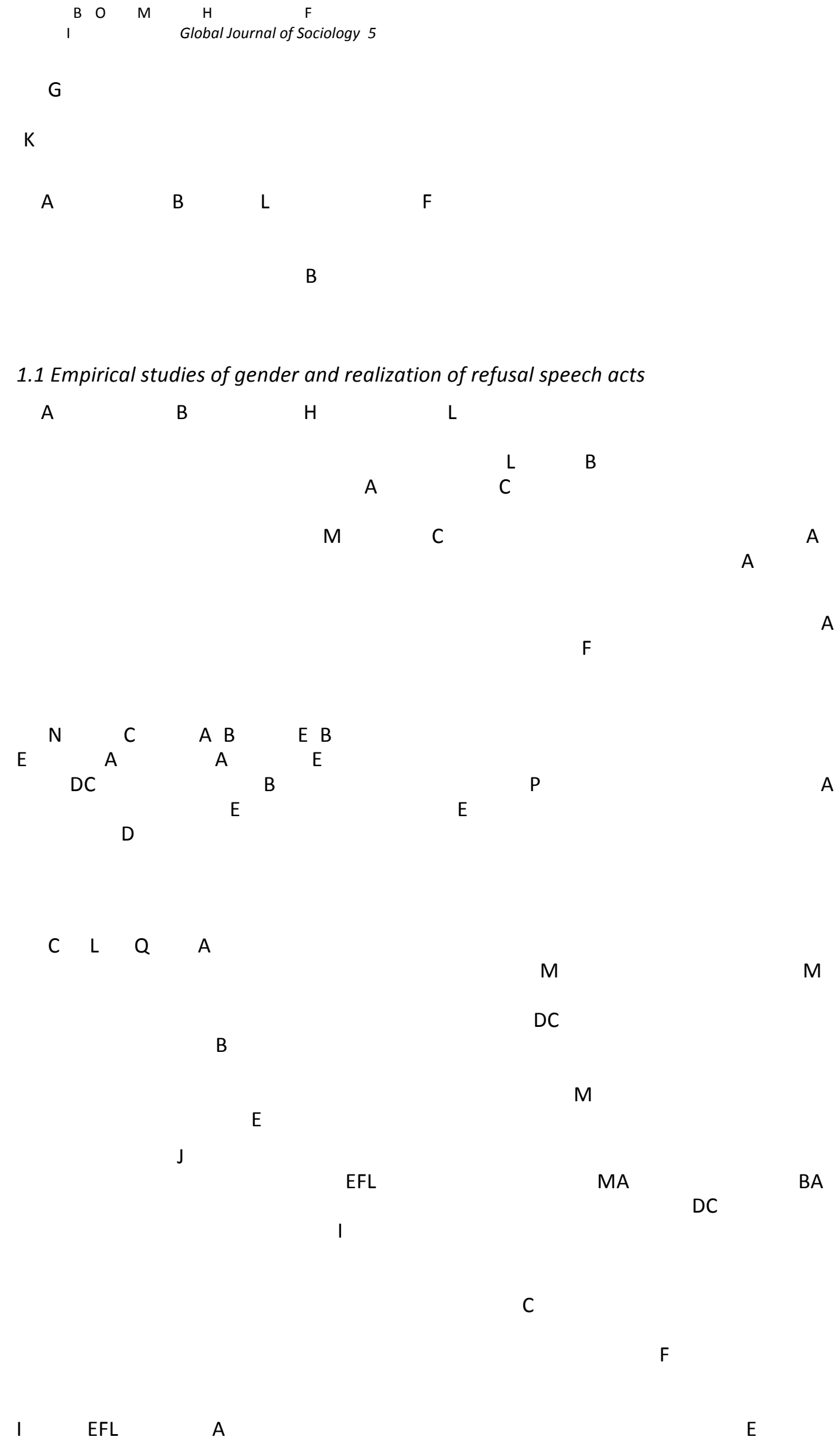


$\begin{array}{lllll}B & \mathrm{O} & \mathrm{M} & \mathrm{H} & \mathrm{F}\end{array}$

I Global Journal of Sociology 5

A A

G

A

B

1.2 Research Questions

D

D

2. Method

2.1 Participants

$\begin{array}{lll}\text { I } & \text { E } & \\ & & \end{array}$

2.2 Instrument

C

A

DC

$$
\text { C }
$$$$
\text { B }
$$

$B$

C

A

C

C

A $\quad$ C

A

DC

\subsection{Procedure}

$\mathrm{P}$

E

IND

A

A

I

0 
$\begin{array}{lllll}B & \mathrm{O} & \mathrm{M} & \mathrm{H} & \mathrm{F}\end{array}$

I Global Journal of Sociology 5

2.4 Coding the refusal strategies

B

I D : No; I can't do it. D

II I I'm sorry; I should pick up my son at the airport. IND

III A I'd love to come, but I can't; A

M I

IND $\quad$ IO

N I

2.5 Data collection

$\mathrm{N}$

DC

DC

2.6 Data analysis

A

$\mathrm{P}$

3. Results

I EFL

C 
$\begin{array}{lllll}\text { B } & \mathrm{O} & \mathrm{M} & \mathrm{H} & \mathrm{F}\end{array}$

I Global Journal of Sociology 5

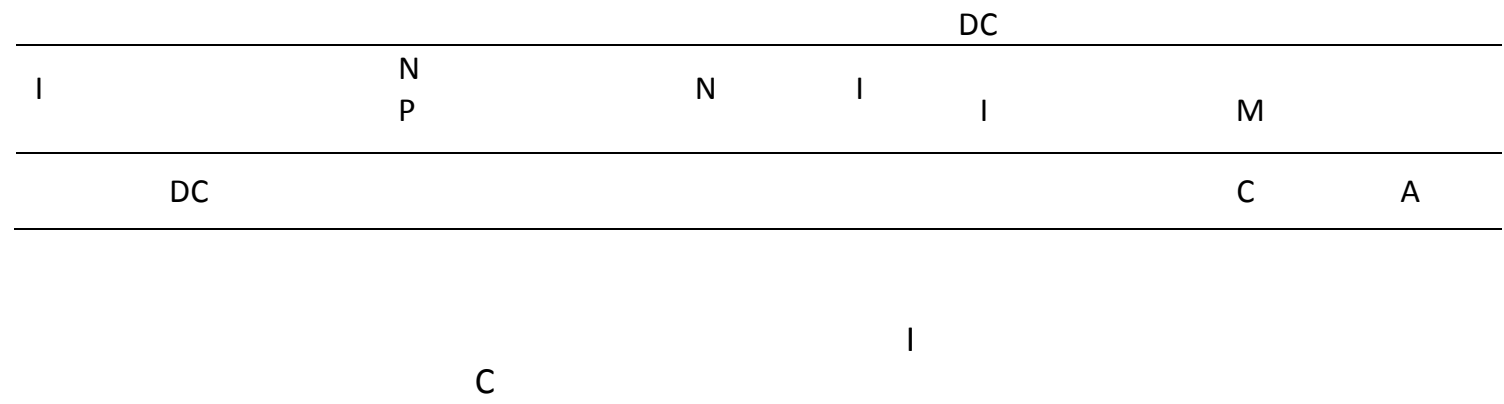


$\begin{array}{ccccc}\text { B } & \mathrm{O} & \mathrm{M} & \mathrm{H} & \mathrm{F} \\ \text { I } & & & \text { Global Journal of Sociology } 5\end{array}$

\begin{tabular}{lllll} 
& & $p$ & $p$ & \\
& & & & \\
& & & & \\
& & & & \\
\hline & & & & \\
\hline $\mathrm{P}$ & $\mathrm{C}$ & $\mathrm{A}$ & $\mathrm{A}$ \\
\hline $\mathrm{N}$ & $\mathrm{C}$ & & \\
\hline
\end{tabular}

F

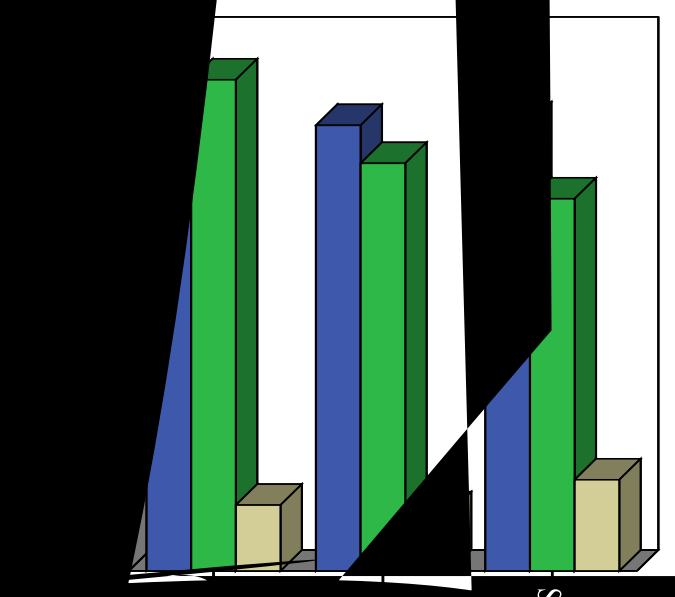




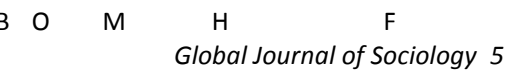

$\mathrm{F}$

\begin{tabular}{llll}
$F$ & $P$ & & \\
& & & $A$ \\
\hline & & & \\
\hline & $C$ & $G$
\end{tabular}

G

M

C

G

C
A D

D

I

A

A

M

\begin{tabular}{lll}
$M$ & $C$ & $G$ \\
& & \\
& $C$ & $G$ \\
\hline
\end{tabular}

F P

A

M

I $\quad$ D

D

I




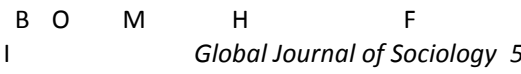

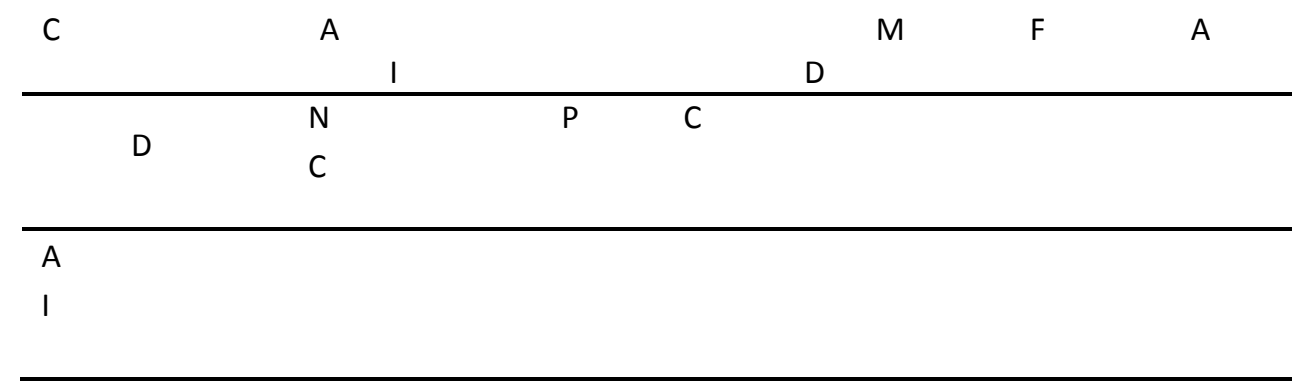

F
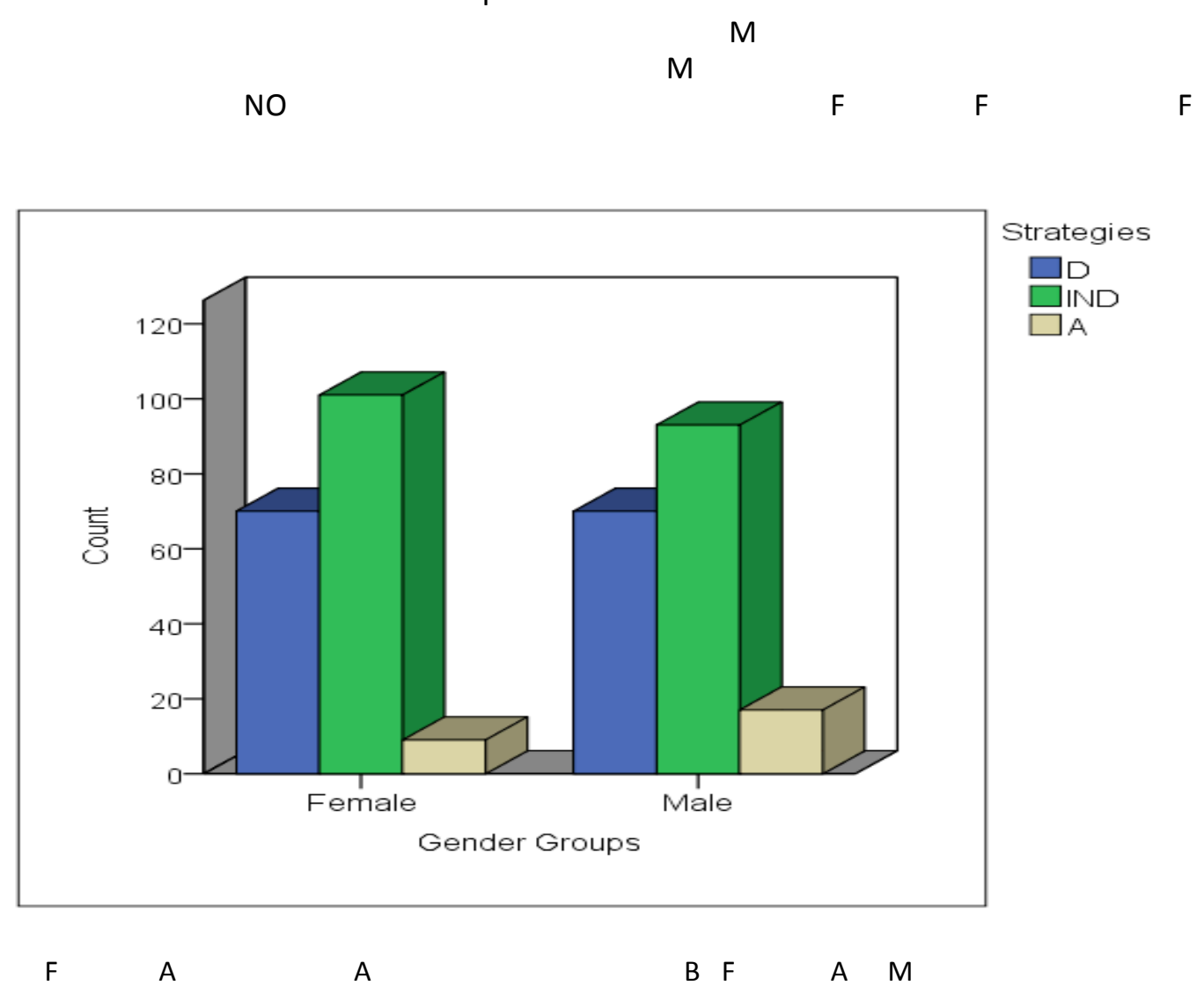


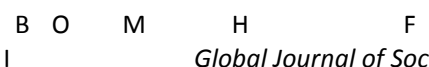

I Global Journal of Sociology 5
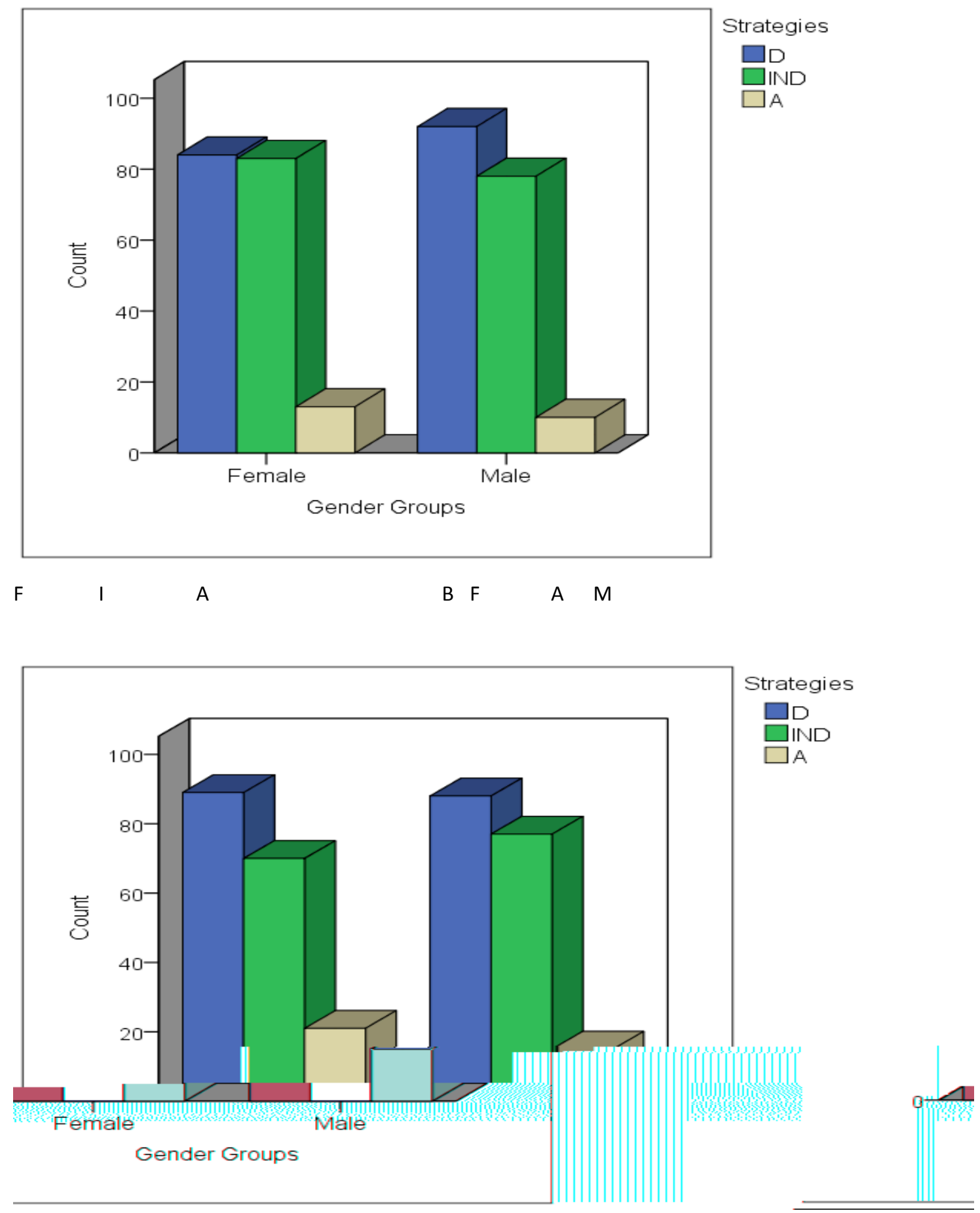

F

\section{Discussion}

D

I EFL 
$\begin{array}{lllll}\text { B } & \mathrm{O} & \mathrm{M} & \mathrm{H} & \mathrm{F} \\ \mathrm{I} & & & \text { Global Journal of Sociology } 5\end{array}$

G

\section{P}

C

IND

\section{Conclusion and Recommendations}

A

A H

EFL

L

$\mathrm{L}$

\section{$\mathrm{L}$}

$\mathrm{FL}$

M

EFL

EFL

I EFL

EFL

\section{Acknowledement}

M O

D B

D 

B $O \quad M$
$\mathrm{H}$
Global Journal of Sociology 5

\section{References}
A $\mathrm{J} L \quad$ How to do things with words $\mathrm{L}$
A A
M A C
O
P Theory and Practice in Language Studies
$\begin{array}{llllllll}B & \mathrm{~B} & \mathrm{O} & \mathrm{M} & \mathrm{F} & \mathrm{A} & \mathrm{H} & \mathrm{J}\end{array}$
AMP
C
Molecular Endocrinology 4
B D
Pragmatics, 19,
B P L
$\mathrm{L} \quad \mathrm{P}$
Journal of
Questions on politeness: Strategies in social interaction $\mathrm{P}$
B P L P C Politeness: Some universals in language usage. C C
C L $\quad$ Q $\quad A$
$\begin{array}{llll} & I & E & E\end{array}$
A
F $M$
J C GEMA Online ${ }^{T M}$ Journal of Lang GEMA Online ${ }^{T M}$ Journal of Language Studies Volume 11(3).
E B $M$
$\mathrm{F}$ Journal of Pragmatics, 38
A
C I
MA E L
C
TESL-EJ
G $\quad \mathrm{M} \quad \mathrm{H} \quad \mathrm{N} \quad$ Interlanguage refusals: A cross-cultural study of Japanese-English. $\mathrm{N}$
G E Interaction ritual: Essays on face-to-face behavior. N
G$$
\text { Language Studies, }
$$
A
A C
ACADEM P BLI HE M

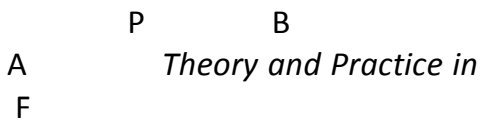

$\mathrm{H} \quad \mathrm{J}$ Women, men and politeness. $\mathrm{L} \quad \mathrm{L}$

K G B

Themes in SLA research. AILTA. Review

P

C Language and woman's place. N

$\mathrm{H}$

$\mathrm{L}$ C B $\mathrm{M} \mathrm{J}$

A

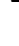

N

Language Sciences, 18

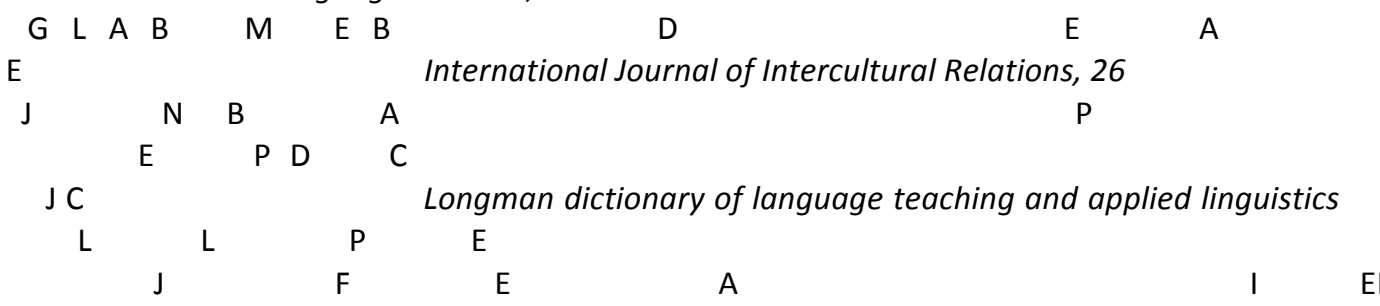

L

Cross-Cultural Communication 7 $\mathrm{E}$

\section{C}

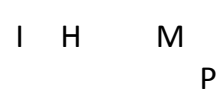

B L M

E JALT Journal, 8,

Speech Act Set of Refusal and Comparison of Native and Non-Native English Speakers' Production

A

D You just don't understand: Men and women in conversation $\mathrm{N}$ M C

P N

Cross - Cultural Communication, 7 
$\begin{array}{llll}B & \mathrm{M} & \mathrm{H} & \mathrm{F}\end{array}$

I Global Journal of Sociology 5

C A

Working Papers in ESL, 15

$\mathrm{L} H \mathrm{H}$ A

C $\mathrm{E}$

A C

Polyglossia, 18

A

University of Hawai'i

EFL L

P

F

I 